\title{
COMMENT
}

\section{The definition of bronchopulmonary dysplasia: an evolving dilemma}

\author{
John Ibrahim ${ }^{1}$ and Vineet Bhandari ${ }^{2}$
}

The definition of bronchopulmonary dysplasia (BPD) continues to be a challenging one. The evolution of the disease as well as its definition are discussed. Limitations of the current as well as newer suggested definitions of BPD are summarized. Recognition of the variability of the pulmonary phenotype is a critical aspect of the disease that needs to identified, utilizing biomarkers that will potentially better inform the next iteration of the definition of BPD.

Pediatric Research (2018) 84:586-588; https://doi.org/10.1038/s41390-018-0167-9

\begin{abstract}
BACKGROUND
In the United States, bronchopulmonary dysplasia (BPD), the most common chronic lung disease in infants, impacts $10,000-15,000$ infants annually, including $~ 50 \%$ of the infants born with birth weight $<1000 \mathrm{~g}^{1}$ Chronic respiratory and cardiovascular impairments leading to eventual growth failure and neurodevelopmental delay are common among affected neonates. In fact, patients with BPD were found to more likely be hospitalized for wheezing, asthma ${ }^{2}$ and respiratory infections. ${ }^{3}$ In a cohort of school-aged children, born preterm and who had BPD, there was significant impairment in exercise capacity despite evidence of only small airway obstruction and gas trapping. $^{4}$

The effect of BPD extends to short- and long-term economic impact on the society. Hospitalization costs for infants with BPD were 16 times greater than for infants without BPD. ${ }^{5}$ The median cost for neonatal intensive care unit (NICU) hospitalization for neonates with BPD was $\$ 102,000$ (US dollars, 2001) per infant in comparison to hospital costs of $\$ 36,800$ for all neonates born at $<28$ weeks gestation. ${ }^{6}$ BPD also leads to increased parental stress and psychological diseases in the form of depression, anxiety and posttraumatic stress disorder. In a study comparing mothers of very low birth weight (VLBW) infants with BPD with VLBW infants without BPD and term infants until adolescence, mothers of VLBW infants with BPD had increased personal, family and financial stresses. $^{7,8}$
\end{abstract}

\section{BPD DEFINITION: A HISTORICAL PERSPECTIVE}

The definition of BPD has evolved since the 1960s when it was first described. This can be attributed largely to its underlying multifactorial pathophysiology 9,10 that continues to evolve. The changes in its clinical presentation in recent years have rendered most of the old definitions obsolete. Subsequently, the wide spectrum of definitions has led to a lack of uniform diagnostic criteria among neonatologists and a reported wide variation in its incidence among different centers.
In 1962, Boss and Craig et al. reported microscopical findings in infants dying from hyaline membrane disease (HMD) in the first 2 weeks of life. They noted proliferative changes involving the alveolar lining cells in areas adjacent to respiratory bronchioles and periphery of the lungs, associated with thickening of the alveolar walls. ${ }^{11}$ Similar findings were described by Robertson et al. in three children who died of severe HMD at 12-23 days of age. ${ }^{12}$

In 1967, Northway et al. coined the term "bronchopulmonary dysplasia" in a seminal description of clinical, pathologic and radiographic characteristics of lung disease in a cohort of 32 moderate to late preterm infants, with a mean gestational age of 34 weeks and a mean birth weight of $2200 \mathrm{~g}^{13}$

In 1968, Shepard et al. first reported the radiographic findings of pulmonary fibrosis in a series of mechanically ventilated preterm infants at Vanderbilt University Hospital (mean gestational age of 36 weeks). ${ }^{14}$ In their study, Shepard et al. described patchy areas of fibrosis and disruption of alveolar architecture on lung biopsy specimens taken from two of the neonates who had frequent severe episodes of pulmonary overexpansion, with wheezing, rales and rhonchi requiring frequent admissions in the first 2 years of life.

\section{EVOLUTION OF BPD UNDERSTANDING AND LIMITATIONS OF ITS DEFINITIONS}

In 1978, a National Heart, Lung, and Blood Institute (NHLBI) sponsored a workshop that defined BPD as "28 days of oxygen exposure with characteristic radiographic changes". This was reported in $1979 .{ }^{15}$ Shennan et al., a decade later, followed up 119 neonates with birth weights less than $1500 \mathrm{~g}$ for 2 years. ${ }^{16} \mathrm{He}$ reported that oxygen requirement at 28 days of life had a predictive value for abnormal pulmonary findings of only $38 \%$, while $\sim 31 \%$ of the control infants with normal pulmonary function were still receiving oxygen at the time of follow-up. He concluded that "The need for oxygen at 28 days was a good predictor of abnormal findings in infants of 30 weeks' gestational age at birth

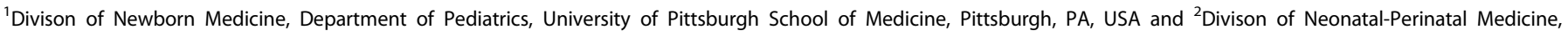
Department of Pediatrics, Drexel University College of Medicine, Philadelphia, PA, USA

Correspondence: Vineet Bhandari (vineet.bhandari@drexel.edu)

Received: 14 August 2018 Accepted: 19 August 2018

Published online: 4 September 2018 
but became increasingly less useful as gestational age decreased." The study reported that irrespective of the gestational age at birth, the oxygen requirement at 36 weeks corrected was a better predictor of abnormal pulmonary outcome, with a predictive value of $63 \%$.

With the improvement in neonatal care and the introduction of gentler ventilation strategies, the use of antenatal steroids and surfactant therapy, the pathophysiology of lung disease in premature infants changed, leading to major differences on the histological level, which in turn changed the management strategies of BPD.

The evolution of understanding the disease process behind the lung injury sustained in preterm infants drew more attention and postulations. Stocker et al. reported that $68 \%$ of the time progressive respiratory failure was the cause of death among 28 infants who died between 3-40 months. ${ }^{17}$ Eighteen percent of these neonates died of pneumonia superimposed on longstanding healed BPD. On the histological level, the study described the pathological features of long-standing healed BPD, for which alveolar septal fibrosis was the main presenting feature. There was a striking difference in the degree of fibrosis between different acini in the same infant. With the degree of fibrosis being relatively uniform within an acinus, an acinus adjacent to it might have no fibrosis or have a milder or more severe degree of fibrosis. The authors concluded that this difference in severity and distribution of fibrosis leading to partial or complete luminal occlusion could be protective to the distal parenchyma against the high pressures and oxygen tension used during ventilation of neonates with severe HMD.

Husain et al. studied a group of neonates divided into three groups, surfactant-treated BPD patients (S-BPD), non-surfactanttreated (NS-BPD) and age-matched controls. ${ }^{18}$ There was less and more diffuse alveolar septal fibrosis in S-BPD than in NS-BPD. There was partial to complete arrest in acinar development (reduced radial alveolar count or RAC and increased mean linear intercept or MLI), of similar severity in S-BPD and NS-BPD neonates. The authors concluded that "the pathology of BPD has changed once again, this time because of the use of surfactant." These histological differences and birth of neonates at an earlier stage of lung development (saccular stage), in addition to the introduction of surfactant, led Jobe to make a distinction between "old" and "new" BPD. ${ }^{19}$ The "New BPD" is seen more in surfactant-treated extremely LBW (ELBW) infants. "New BPD" is due to disruption of lung development, rather than mechanical ventilation and oxygen toxicity. On a histological level, the "New BPD" manifests as decreased septation, alveolar hypoplasia and dysregulated development of pulmonary vasculature. "Old BPD" has abnormal arterial vascularization with obliteration of vessels and pulmonary fibrosis.

Limitations of the National Institutes of Health (NIH) sponsored workshop definition of BPD in 1979 were mainly seen in infants with "New BPD". Most infants with "New BPD" may have intervals of time during the first weeks after birth where they don't require supplemental oxygen. ${ }^{20}$ The definition has resulted in a marked variability in the incidences of BPD among different centers when applied to the same population of premature infants (for 28 days versus at 28 days).

The National Institute of Child Health and Human Development (NICHD), NHLBI and Office of Rare Diseases (ORD), in 2000, proposed the current (NIH consensus) definition of BPD, 21 "Infants born $<32$ weeks, who require supplemental oxygen for at least 28 days and at 36 weeks postmenstrual age (PMA)". The definition further stratified the disease into mild (breathing room air at 36 weeks PMA or discharge, whichever comes first), moderate (need for $<30 \%$ oxygen at 36 weeks PMA or discharge, whichever comes first), severe (need for $\geq 30 \%$ oxygen and /or positive pressure [positive pressure ventilation (PPV) or nasal continuous positive airway pressure (NCPAP)] at 36 weeks PMA or discharge, whichever comes first). Some important differences from earlier definitions were as follows: it was the first time BPD was stratified based on oxygen requirement and type of respiratory support at 36 weeks PMA, and radiographic changes were no longer one of the required criteria.

Despite studies showing the association between the $\mathrm{NIH}$ consensus definition and long-term neurodevelopmental outcomes, ${ }^{22}$ this definition still bears some of the shortcomings: ${ }^{20}$ it is hard to standardize the appropriate oxygen saturation limits for preterm neonates across various centers, as it is still a hot topic for research, ${ }^{23-25}$ and the use of corrected gestational age at 36 weeks PMA means that the more mature infants will be labeled as having BPD with a much shorter duration of oxygen exposure. One of the disadvantages of this definition is that it tends to classify a chronic disease based on oxygen exposure at a specific time point. One of the other problems with this consensus definition is not considering factors that can influence oxygen saturation; for example, altitude or gas exchange (due to mode of ventilation, presence of airway obstruction because of tracheomalacia) and use of certain medications (diuretics, steroids and pulmonary vasodilators).

In an effort to minimize the variability of BPD diagnosis across different centers, Walsh et al. $^{26}$ proposed the "physiologic" definition of BPD through an "oxygen reduction test". Neonates at $36 \pm 1$ weeks receiving $\leq 30 \%$ oxygen underwent a $2 \%$ stepwise reduction in supplemental oxygen every $10 \mathrm{~min}$ to room air with continuous observation and oxygen saturation monitoring. Failure was defined as oxygen saturation of $80-87 \%$ for $5 \mathrm{~min}$ or $<80 \%$ for 1 minute. The infant was monitored for $60 \mathrm{~min}$ in room air and assigned as having no BPD when all saturations exceeded $88 \%$ during that time. Rapid pass was defined as successful weaning to room air with all saturations $\geq 96 \%$ for $15 \mathrm{~min}$. The application of this new physiologic definition of BPD reduced the rates of BPD from $35 \%$ by clinical definition to $25 \%$ by "physiologic" definition across 17 centers of the neonatal research network (NRN). ${ }^{27}$ The study acknowledged that significant variation in the rates of BPD remained among centers.

Not only has the scientific community struggled to standardize the definition of BPD, but also there is significant controversy regarding the accurate stratification of the disease based on severity. BPD pathogenesis and prevention has been the spotlight of many research trials. The respiratory care for neonates with chronic lung disease has largely focused on the early neonatal period. However, despite improvement in neonatal practices, a small cohort of extremely preterm infants continue to develop severe BPD (sBPD) requiring considerable respiratory support and high oxygen concentrations. In fact, a recent paper shed light on the necessity to further categorize the phenotypes of severe BPD in the $\mathrm{NIH}$ consensus definition, based on whether neonates have persistent oxygen requirement and/or need for CPAP or high flow nasal cannula (HFNC) at 36 weeks PMA (type 1 sBPD) or they are ventilator-dependent and have frequent severe complications, including pulmonary hypertension, poor growth and poor neurodevelopmental outcomes (type 2 sBPD). ${ }^{28}$ The rationale behind this classification according to the authors of the "BPD collaborative" was that the management strategies of chronic lung disease vary from acute respiratory failure in many aspects, including pulmonary toilet and ventilation approach. Physicians, particularly the newer-generation neonatologists, may be less comfortable with ventilation strategies of infants with SBPD, especially with it becoming less common with the introduction of surfactant for respiratory distress syndrome. On the patient level, the prolonged hospital care of these chronic neonates, with frequent changes of the physicians on service, and the high turnover of the subspecialists and nursing staff, may affect the quality of care and consistency of plans of management as well as increase the level of frustration in their already anxious parents. 


\section{CURRENT CHALLENGES AND PROSPECTS TO IMPROVE THE DEFINITION OF BPD}

The statement by McEvoy et al, ${ }^{29}$ accurately delineates the evolving dilemma of defining BPD: "Strategies for primary prevention are stymied by methodologic obstacles, not the least of which is the inherent shortcoming of defining a disease by its treatment and the use of a definition that provides no information about pathophysiology, disease progression, or phenotype variability".

Recognition of the pulmonary phenotypes of BPD based on clinical and biomarker stratification should be the next step in refining the definition of $B P D$. While the recent summary and suggestion of a NICHD workshop further classifies BPD based on the degrees of respiratory support, ${ }^{30}$ it does not overcome the inherent limitation of utilizing a therapeutic approach to define a disease with a variable pulmonary phenotype, among other concerns. ${ }^{31}$ In a recent retrospective analysis, the use of oxygen and respiratory support at 40 weeks' PMA was identified as the best predictor for serious respiratory morbidity and had a good ability to predict neurodevelopmental outcomes at 18-21 months corrected age. ${ }^{32}$

We would suggest that an improved delineation of the pulmonary phenotypes of BPD can be achieved by utilizing biomarkers. While circulating cytokine patterns may be potentially useful, they may not necessarily reflect what goes on in the lung. ${ }^{33}$ Getting access to the lung compartment via tracheal aspirates and utilizing an "omics" approach may perhaps allow us a better definition of BPD. ${ }^{34,35}$

\section{CONCLUSION}

BPD is a challenging disease with long-term lasting effects on the health of our premature survivors, their parents and society. Yet, despite all this interest from the research community to prevent and combat its clinical course, there has not been a standard definition that best describes the disease. We believe that the future results of ongoing studies might make it feasible to define BPD based on accurate clinical pathophysiology and biomarker identification, rather than interventions. It is important to highlight the fact that since BPD is an independent predictor of long-term pulmonary and neurological outcomes, the earlier a definitive recognition is made of this disease, the more likely will it be to develop therapeutic strategies to prevent the mortality and morbidity associated with this chronic life-long condition.

\section{ADDITIONAL INFORMATION}

Competing interests: The authors declare no competing interests.

Publisher's note: Springer Nature remains neutral with regard to jurisdictional claims in published maps and institutional affiliations.

\section{REFERENCES}

1. Stoll, B. J. et al. Neonatal outcomes of extremely preterm infants from the NICHD Neonatal Research Network. Pediatrics 126, 443-456 (2010).

2. Urs, R., Kotecha, S., Hall, G. L. \& Simpson, S. J. Persistent and progressive longterm lung disease in survivors of preterm birth. Paediatr. Respir. Rev. (2018). pii: S1526-0542(18)30052-6. https://doi.org/10.1016/j.prrv.2018.04.001.

3. Korhonen, P., Laitinen, J., Hyodynmaa, E. \& Tammela, O. Respiratory outcome in school-aged, very-low-birth-weight children in the surfactant era. Acta Paediatr. 93, 316-321 (2004).

4. Smith, L. J., van Asperen, P. P., McKay, K. O., Selvadurai, H. \& Fitzgerald, D. A. Reduced exercise capacity in children born very preterm. Pediatrics 122, e287-e293 (2008).

5. Russell, R. B. et al. Cost of hospitalization for preterm and low birth weight infants in the United States. Pediatrics 120, e1-e9 (2007).
6. Wannasiri Lapcharoensap, M., Lee, H. C., Nyberg, A. \& Dukhovny, D. Health care and societal costs of bronchopulmonary dysplasia. Neoreviews 19, e211-e223 (2018).

7. Singer, L. T. et al. Parenting very low birth weight children at school age: maternal stress and coping. J. Pediatr. 151, 463-469 (2007).

8. Singer, L. T. et al. Longitudinal predictors of maternal stress and coping after very low-birth-weight birth. Arch. Pediatr. Adolesc. Med. 164, 518-524 (2010).

9. Kalikkot Thekkeveedu, R., Guaman, M. C. \& Shivanna, B. Bronchopulmonary dysplasia: a review of pathogenesis and pathophysiology. Respir. Med. 132, 170-177 (2017).

10. Jobe, A. H. Mechanisms of lung injury and bronchopulmonary dysplasia. Am. J. Perinatol. 33, 1076-1078 (2016).

11. Boss, J. H. \& Craig, J. M. Reparative phenomena in lungs of neonates with hyaline membranes. Pediatrics 29, 890-898 (1962).

12. Robertson, B., Tunell, R. \& Rudhe, U. Late stages of pulmonary hyaline membranes of the newborn. Acta Paediatr. 53, 433-446 (1964).

13. Northway, W. H. Jr., Rosan, R. C. \& Porter, D. Y. Pulmonary disease following respirator therapy of hyaline-membrane disease. Bronchopulmonary dysplasia. N. Engl. J. Med. 276, 357-368 (1967).

14. Shepard, F. M., Johnston, R. B. Jr, Klatte, E. C., Burko, H. \& Stahlman, M. Residual pulmonary findings in clinical hyaline-membrane disease. N. Engl. J. Med. 279, 1063-1071 (1968).

15. National Heart, Lung, and Blood Institute, National Institutes of Health. Workshop on bronchopulmonary dysplasia. Sponsored by the Division of Lung Diseases. J. Pediatr. 95, 815-920 (1979).

16. Shennan, A. T., Dunn, M. S., Ohlsson, A., Lennox, K. \& Hoskins, E. M. Abnormal pulmonary outcomes in premature infants: prediction from oxygen requirement in the neonatal period. Pediatrics 82, 527-532 (1988).

17. Stocker, J. T. Pathologic features of long-standing "healed" bronchopulmonary dysplasia: a study of 28 3- to 40-month-old infants. Hum. Pathol. 17, 943-961 (1986).

18. Husain, A. N., Siddiqui, N. H. \& Stocker, J. T. Pathology of arrested acinar development in postsurfactant bronchopulmonary dysplasia. Hum. Pathol. 29, 710-717 (1998)

19. Jobe, A. H. The new BPD: an arrest of lung development. Pediatr. Res. 46, 641-643 (1999).

20. Bancalari, E. \& Claure, N. Definitions and diagnostic criteria for bronchopulmonary dysplasia. Semin. Perinatol. 30, 164-170 (2006).

21. Jobe, A. H. \& Bancalari, E. Bronchopulmonary dysplasia. Am. J. Respir. Crit. Care Med. 163, 1723-1729 (2001).

22. Ehrenkranz, R. A. et al. Validation of the National Institutes of Health consensus definition of bronchopulmonary dysplasia. Pediatrics 116, 1353-1360 (2005).

23. Network SSGotEKSNNR, Carlo, W. A. et al. Target ranges of oxygen saturation in extremely preterm infants. N. Engl. J. Med. 362, 1959-1969 (2010)

24. Group BIUKC, Group BIAC, Group BINZC et al. Oxygen saturation and outcomes in preterm infants. N. Engl. J. Med. 368, 2094-2104 (2013)

25. Nelin, L. D. \& Bhandari, V. How to decrease bronchopulmonary dysplasia in your neonatal intensive care unit today and "tomorrow". F1000Research 6, 539 (2017).

26. Walsh, M. C., Wilson-Costello, D., Zadell, A., Newman, N. \& Fanaroff, A. Safety, reliability, and validity of a physiologic definition of bronchopulmonary dysplasia. J. Perinatol. 23, 451-456 (2003).

27. Walsh, M. C. et al. Impact of a physiologic definition on bronchopulmonary dysplasia rates. Pediatrics 114, 1305-1311 (2004).

28. Abman, S. H. et al. Interdisciplinary care of children with severe bronchopulmonary dysplasia. J. Pediatr. 181, 12-28 e11 (2017).

29. McEvoy, C. T. et al. Bronchopulmonary dysplasia: NHLBI Workshop on the Primary Prevention of Chronic Lung Diseases. Ann. Am. Thorac. Soc. 11(Suppl 3), S146-S153 (2014).

30. Higgins, R. D. et al. Bronchopulmonary dysplasia: Executive Summary of a Workshop. J. Pediatr. 197, 300-308 (2018).

31. Jensen, E. A. \& Wright, C. J. Bronchopulmonary dysplasia: the ongoing search for one definition to rule them all. J. Pediatr. 197, 8-10 (2018).

32. Isayama, T. et al. Revisiting the definition of bronchopulmonary dysplasia: effect of changing panoply of respiratory support for preterm neonates. JAMA Pediatr. 171, 271-279 (2017).

33. D'Angio, C. T. et al. Blood cytokine profiles associated with distinct patterns of bronchopulmonary dysplasia among extremely low birth weight infants. J. Pediatr. 174, 45-51 e45 (2016).

34. Lal, C. V. \& Ambalavanan, N. Cellular and humoral biomarkers of bronchopulmonary dysplasia. Early Hum. Dev. 105, 35-39 (2017).

35. Piersigilli, F. \& Bhandari, V. Biomarkers in neonatology: the new "omics" of bronchopulmonary dysplasia. J. Matern. Fetal Neonatal Med. 29, 1758-1764 (2016). 